\title{
ESTIMACIÓN DE BIOMASA Y CARBONO EN UN BOSQUE DE Abies religiosa
}

\author{
BIOMASS AND CARBON ESTIMATION IN AN Abies religiosa FOREST
}

\author{
${ }^{1}$ Dulce Ma. Avendaño Hernandez, Miguel Acosta Mireles ${ }^{2 *}$, Fernando Carrillo Anzures ${ }^{3}$ y Jorge D. \\ Etchevers Barra ${ }^{4}$
}

\begin{abstract}
${ }^{1}$ Comisión Nacional Forestal, Subgerencia Estatal Morelos. Río Balsas No. 31. 62130, Cuernavaca Morelos. ${ }^{2,3}$ Campo Experimental Valle de México, Instituto Nacional de Investigaciones Forestales, Agrícolas y Pecuarias. Km. 18.5 Carr. Los Reyes-Lechería. 56230, Chapingo, Edo. de México. Tel. (01)(595)9542877 Ext. 142. ${ }^{4}$ Colegio de Postgraduados. Km.36.5 Carr. México-Texcoco, 56239, Montecillo, Texcoco Edo. de México.
\end{abstract}

\section{RESUMEN}

Se generaron dos ecuaciones para determinar biomasa y carbono en Abies religiosa (H.B.K.) Schl. et Cham. Tales ecuaciones son de la forma $Y=b X^{k}$, donde $Y$ es el contenido de biomasa o de carbono, $y$ $\mathrm{X}$ el diámetro normal (DN). Para estimar los valores $\boldsymbol{b}$ y $\boldsymbol{k}$ del modelo se utilizaron datos de 26 árboles de un área en aprovechamiento forestal, con diámetro normal entre 6.5 y $79 \mathrm{~cm}$ del predio " $\mathrm{El}$ Innominado" en San Felipe Hidalgo Nanacamilpa, Tlaxcala, México. A cada árbol se le determinó la biomasa de sus componentes (fuste, ramas y follaje); de este material se seleccionaron al azar 10 submuestras de fuste, 10 de ramas y 6 de follaje, y todas se analizaron para determinar su concentración de carbono. La concentración de carbono en el fuste fue de $\mathbf{4 6 . 6} \%$, en las ramas fue de $46 \%$ y en follaje $46.1 \%$, y la concentración promedio de carbono por árbol fue de $46.5 \%$. El mayor porcentaje de biomasa (84.5\%) se encontró en el fuste incluido el tocón, mientras que en las ramas y el follaje hubo 6.9 y $8.6 \%$, respectivamente. Con la biomasa y el DN de los 26 árboles se obtuvo el ajuste de los parámetros $b$ y $k$ del modelo propuesto $\left(\mathrm{B}=\mathbf{0 . 0 7 1 3} \mathrm{DN}^{2.5104}\right)$. Para establecer las variables del modelo predictivo de carbono acumulado por árbol (CAA) en función del DN, se usó un procedimiento idéntico al anterior, cuyo resultado fue $\mathrm{CAA}=\mathbf{0 . 0 3 3 2} \mathrm{DN}^{2.5104}$. Los parámetros para ambas ecuaciones fueron estimados con un coeficiente de determinación $\left(\mathbf{R}^{2}\right)$ de 0.99 .

Palabras clave: Abies religiosa, almacén de carbono, biomasa, ecuaciones alométricas.

\section{SUMMARY}

Two equations were generated to determine biomass and carbon in Abies religiosa (H.B.K.) Schl. et Cham. These equations were of the form $\mathrm{Y}=\boldsymbol{b} \mathrm{X}^{k}$, where $\mathrm{Y}$ is the content of biomass or carbon, and $X$ the diameter at the breast height (DBH). In order to estimate the values $b$ and $k$, date from 26 trees of an area under forest management were used, with basal a diameter between 6.5 and $79 \mathrm{~cm}$ at the stand called "El Innominado" at San Felipe Hidalgo, in Nanacamilpa County, Tlaxcala, México. The biomass of each component of every tree was determined (logs, branches and foliage). Ten random subsamples of $\mathrm{log}$, ten of branches and six of foliage were analyzed to determine carbon content. The carbon concentration in $\log$ was $46.6 \%$, in branches was $46 \%$ and $46.1 \%$ in the foliage. The carbon concentration average by tree was $46.5 \%$. The greater percentage of biomass $(84.5 \%)$ was found in the $\log$ including the stump, whereas in the branches in and the foliage it was 6.9 and 8.6\%, respectively. The adjustment of parameters $b$ and $k$ of the proposed model were obtained with the biomass and the basal diameter of the 26 trees $\left(B=0.0713 \mathrm{DN}^{2.5104}\right)$. The variables of the predictive model of accumulated carbon by tree (CAA) based on the Dbh were determined by an identical procedure, and the result was CAA $=0.0332 \mathrm{DN}^{2.5104}$. The parameters for both equations had a coefficient of determination $\left(R^{2}\right)$ of 0.99 .

Index words: Abies religiosa, carbon stock, biomass, alometric ecuations.

\section{INTRODUCCIÓN}

La concentración de bióxido de carbono en la atmósfera ha aumentado de $280 \mathrm{ppm}$ (i.e., $280 \mathrm{~mL} \mathrm{~L}^{-1}$ ) en el año 1750 a 379 ppm en el 2004, y continúa incrementando a una tasa anual mayor a $1 \mathrm{ppm}$ (Castro et al., 2002; Epstein y Rogers, 2004; NETL, 2004). Al evaluar la vulnerabilidad de ecosistemas forestales por medio de la aplicación de tres modelos de cambio climático, Villers y Trejo (2000) encontraron que los bosques templados fríos y semicálidos son los más sensibles al cambio climático y tenderían a desaparecer al incrementarse la temperatura. Otras implicaciones que aún no se han evaluado pero que podrían suceder por el aumento de la temperatura en la tierra como efecto del aumento de los gases de efecto invernadero (GEI), es que podrían aumentar algunas poblaciones de especies dañinas como plagas o enfermedades en perjuicio de algunos cultivos.

Para reducir la emisión de $\mathrm{CO}_{2}$ a la atmósfera se han implementado procesos de origen biótico y abiótico, como 
el aprovechamiento de la capacidad de fijación de carbono (proceso biótico) de los ecosistemas forestales, que aún es desconocida y no se cuenta con procedimientos definidos para su estimación. Esta capacidad varía en función de la composición florística, la edad y la densidad de la población en cada estrato de la comunidad vegetal (Schulze et al., 2000), pero aún falta mucho por investigar al respecto.

Una alternativa biótica para reducir la cantidad de $\mathrm{CO}_{2}$ en la atmósfera es absorber parte de este gas por la vegetación mediante el proceso de la fotosíntesis, y que además las plantas funcionen como almacén de $\mathrm{C}$ en forma de biomasa vegetal y que después se convierte parcialmente en materia orgánica. Conocer la cantidad de biomasa de las especies arbóreas es necesario para estimar la cantidad de carbono que captura un bosque, proceso que representa actualmente un servicio ambiental y, por tanto, constituye una alternativa para el manejo de recursos naturales encaminada al desarrollo sustentable, y tambien para la obtención de algún bien para los propietarios de tales recursos.

El análisis dimensional o alometría es el estudio del cambio de proporción de varias partes de un organismo como resultado de su crecimiento, que en árboles requiere contar con funciones matemáticas que estimen la biomasa total con base en las dimensiones de los órganos del árbol (Huxley, 1932). Las relaciones alométricas se han utilizado mucho en especies forestales de clima templadofrío para estimar la biomasa aérea total y el área foliar a partir del diámetro normal o del área de la albura (Grier y Waring, 1974; Geron y Ruark, 1988; Chapman y Gower, 1991; Castellanos et al., 1996), que sirven para relacionar componentes de difícil medición (biomasa, área foliar) con variables más fáciles de medir (altura, diámetro, área basal, área de la albura) (Pastor et al., 1984).

El presente estudio se llevó a cabo con el objetivo de estimar, por medio de ecuaciones alométricas, la biomasa y el carbono de la parte aérea en bosques de Abies religiosa (H.B.K.) Schl. et Cham., en el Estado de Tlaxcala, México.

\section{MATERIALES Y MÉTODOS}

Para generar las ecuaciones alométricas fue necesario hacer un muestreo destructivo, en el que los árboles se derribaron y sus partes (fuste o tallo, ramas y follaje) fueron pesadas por separado. Por ello este estudio se llevó a cabo en un predio sometido a aprovechamiento forestal.

Se seleccionaron 26 árboles de Abies religiosa con fuste recto y libre de daños, y se tuvo cuidado de incluir la mayoría de categorías diamétricas presentes. Previo al derribo, a cada árbol se le midió el diámetro normal (DN); posteriormente se desramó y el fuste se seccionó en trozas comerciales de 2.55 y $1.25 \mathrm{~m}$ de longitud. De cada troza del fuste se obtuvo una rodaja de aproximadamente $5 \mathrm{~cm}$ de espesor en la parte de mayor diámetro (Figura 1). Las ramas fueron separadas del follaje y luego pesadas, luego de haber obtenido por lo menos cuatro muestras de cada componente que se identificaron con el número de muestra y el número de árbol correspondiente. El peso fresco de cada componente (trozas, ramas y follaje) y su respectiva muestra se determinó in situ para evitar la pérdida de humedad; el peso se midió con una báscula de reloj de 200 $\mathrm{kg}$ de capacidad o una de $15 \mathrm{~kg}$ para muestras de menor tamaño.

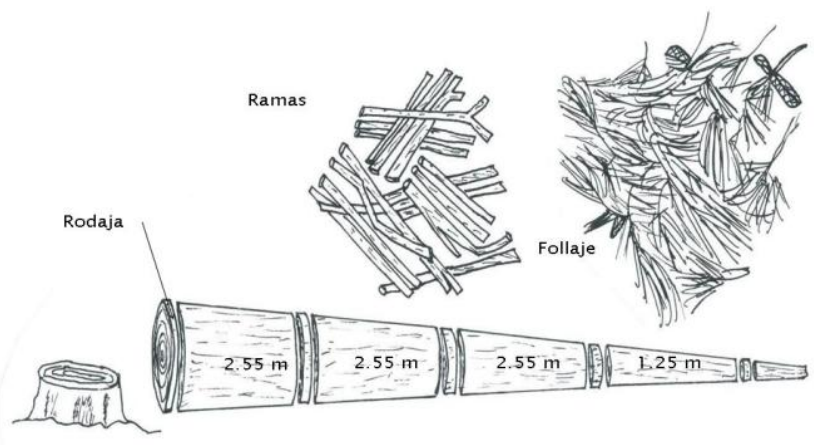

Figura 1. Troceo del arbolado y obtención de rodajas, muestras de ramas y follaje (Tomado de Díaz, 2005).

En total se obtuvieron 406 rodajas de fuste, 77 muestras de ramas y 78 de follaje. Debido al gran volumen de estas muestras, el secado se hizo en un microtúnel, a manera de estufa solar. Este microtúnel consistió en una estructura con cubierta de plástico de $3 \mathrm{~m}$ de ancho y $8 \mathrm{~m}$ de largo, dentro del cual se colocó el material sobre una rejilla de alambre para favorecer la circulación del aire, hasta alcanzar el peso constante (peso seco).

Los árboles más pequeños (de menos de $10.0 \mathrm{~cm}$ de DN) se cortaron y sin ser divididos en partes fueron transportados hasta el lugar en donde se secó todo el material y ahí se pesaron después de secos, para obtener directamente su biomasa.

Para determinar la pérdida de humedad de todas las partes se registró el peso semanal de algunas muestras, las cuales se pesaron cuando el peso se mantuvo constante. Debido a que no se puede obtener un secado total por efecto de la humedad relativa, nueve de ellas se secaron en 
estufa; una vez determinado así su porcentaje de humedad éste se descontó al peso de cada muestra contenida en el microtúnel. Con el peso seco se determinó la relación entre el peso seco y peso fresco de las rodajas, las ramas y el follaje, y los coeficientes se multiplicaron por su respectivo peso fresco para determinar la biomasa de fuste, ramas y follaje de cada árbol. La suma de la biomasa del fuste (trozas y tocón) y de la copa (ramas y follaje) conformó la biomasa aérea total de cada árbol (Acosta et al., 2002).

Una vez obtenidos los datos de biomasa aérea total y el diámetro normal de todos los árboles muestreados, se ajustó el modelo matemático expresado por la Ecs. (1) y (2), en su forma potencial y logaritmica, respectivamente. El Modelo 1 fue ajustado por mínimos cuadrados con el propósito de obtener los valores de los parámetros $b$ y $k$ que representan la ordenada al origen y la pendiente, del modelo de regresión. Este modelo se ha empleado en varios estudios para determinar la biomasa aérea de árboles (Acosta et al., 2002; Figueroa et al., 2005; Gayoso, 2002).

$$
\mathrm{Y}=b \mathrm{X}^{k}
$$

donde $Y$ es la biomasa aérea $(\mathrm{kg}), X$ es el diámetro normal (cm), $b$ y $k$ son los parámetros de la función.

La expresión del Modelo 1 en su forma lineal mediante transformación logarítmica, es la siguiente

$$
\operatorname{Ln}(Y)=\ln (b)+\mathrm{k} \ln (X) \ldots \ldots
$$

donde $Y, X, b$ y $k$ tienen el mismo significado que en la Ec. $1, \mathrm{y} \ln (Y), \ln (b)$ y $\ln (x)$ son los logaritmos naturales de $Y, b$ y $X$, respectivamente.

\section{Determinación del contenido de carbono}

De los 26 árboles seleccionados para la derminación de biomasa se seleccionaron al azar 10 rodajas, 10 muestras de ramas y 6 muestras de follaje para determinar el porcentaje de carbono en cada uno de estos componentes.

De las rodajas se tomaron submuestras de aproximadamente $25 \mathrm{~g}$ y se obtuvieron de forma tal que contuvieran la misma proporción de duramen, albura y corteza, como se muestra en la Figura 2. Cada muestra se molió y mezcló, y en laboratorio se determinó el contenido de carbono en mediante un analizador de carbono TOCV csn® U.S.A.

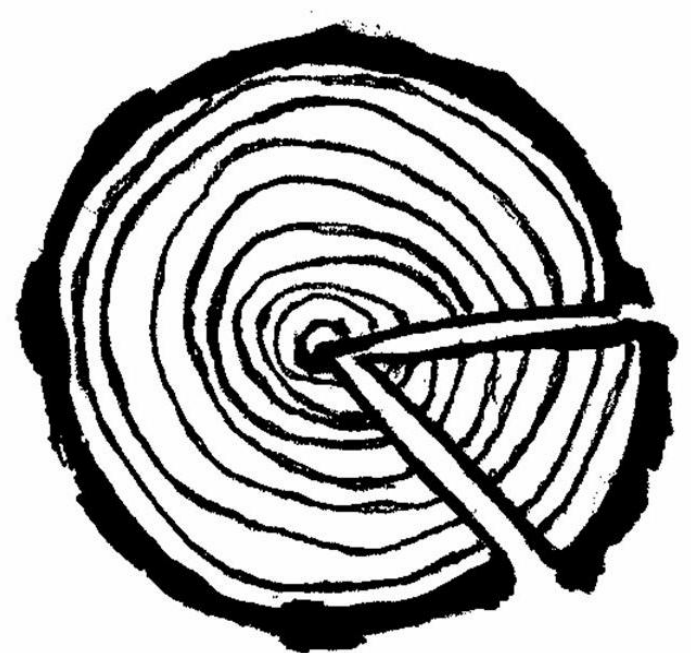

Figura 2. Submuestra tomada de las rodajas para determinar carbono.

Para estimar el contenido de carbono de los 26 árboles muestreados, primero se calculó el porcentaje promedio de las 26 submuestras a las que se les determinó el carbono directamente en el laboratorio; el contenido de carbono en la biomasa de cada árbol se estimó con la siguiente expresión:

$$
\mathrm{Ct}=\mathrm{B} \times \mathrm{FC}
$$

Donde: $\mathrm{Ct}$ es el contenido total de carbono, $\mathrm{B}$ es la biomasa total de cada árbol $(\mathrm{kg})$, y $\mathrm{FC}$ es el factor de carbono determinado en laboratorio.

El contenido del carbono de cada árbol fue la suma del carbono del fuste, ramas y follaje, y para obtener la ecuación alométrica para estimar el carbono se empleó el modelo alométrico que relaciona el contenido de carbono con el diámetro normal de cada árbol.

\section{RESULTADOS Y DISCUSIÓN}

El diámetro normal (DN) de los árboles muestreados varió de 5.7 a $79 \mathrm{~cm}$, con alturas entre 6.8 y $45.5 \mathrm{~m} \mathrm{y}$ biomasa de 5.39 a $3600.77 \mathrm{~kg}$. Las características dasométricas, peso fresco, biomasa y el porcentaje de humedad de cada uno de los 26 árboles muestreados, se muestran en el Cuadro 1. 
Cuadro 1. Características dasométricas de los árboles de Abies religiosa muestreados para generar la ecuación alométrica.

\begin{tabular}{|c|c|c|c|c|c|c|}
\hline Árbol & $\mathrm{DN}(\mathrm{cm})$ & $\mathrm{CD}(\mathrm{cm})$ & Altura (m) & Peso fresco $(\mathrm{kg})$ & Biomasa $(\mathrm{kg})$ & Humedad $(\%)^{\dagger \dagger}$ \\
\hline 1 & 6.5 & 5 & 7.91 & + & 7.28 & $\dagger$ \\
\hline 2 & 5.7 & 5 & 6.79 & $\dagger$ & 5.39 & $\dagger$ \\
\hline 3 & 9.7 & 10 & 11.57 & $\dagger$ & 20.89 & $\dagger$ \\
\hline 4 & 9.5 & 10 & 9.86 & $\dagger$ & 17.91 & $\dagger$ \\
\hline 5 & 15 & 15 & 15.78 & 164.38 & 69.48 & 54.96 \\
\hline 6 & 16 & 15 & 11.9 & 186.19 & 76.79 & 56.04 \\
\hline 7 & 21 & 20 & 24.93 & 413.81 & 182.81 & 52.88 \\
\hline 8 & 18 & 20 & 16.88 & 203.53 & 85.68 & 55.11 \\
\hline 9 & 23 & 25 & 17.93 & 398.96 & 165.74 & 55.70 \\
\hline 10 & 25 & 25 & 19.51 & 574.46 & 246.01 & 54.39 \\
\hline 11 & 29 & 30 & 29.42 & 846.86 & 351.63 & 55.71 \\
\hline 12 & 30 & 30 & 21.74 & 899.93 & 343.86 & 59.25 \\
\hline 13 & 34 & 35 & 32.55 & 1431.18 & 623.43 & 53.53 \\
\hline 14 & 34 & 35 & 35.95 & 1686.49 & 797.44 & 49.54 \\
\hline 15 & 40 & 40 & 30.20 & 1530.19 & 714.92 & 50.15 \\
\hline 16 & 47 & 45 & 37.83 & 3187.44 & 1435.88 & 51.91 \\
\hline 17 & 52 & 50 & 37.23 & 3211.09 & 1417.13 & 52.89 \\
\hline 18 & 48 & 50 & 28.64 & 2321.70 & 967.40 & 55.58 \\
\hline 19 & 57 & 55 & 35.85 & 4199.45 & 1879.61 & 52.23 \\
\hline 20 & 54 & 55 & 35.35 & 3131.54 & 1380.30 & 52.95 \\
\hline 21 & 62 & 60 & 38.61 & 5166.19 & 1987.62 & 58.93 \\
\hline 22 & 60 & 60 & 36.08 & 4356.67 & 1995.62 & 51.11 \\
\hline 23 & 62.5 & 65 & 36.18 & 5004.73 & 2183.88 & 53.43 \\
\hline 24 & 63 & 65 & 44.45 & 6087.11 & 2731.10 & 52.10 \\
\hline 25 & 76 & 75 & 45.46 & 7898.12 & 3209.59 & 56.64 \\
\hline 26 & 79 & 80 & 42.45 & 7957.81 & 3600.77 & 51.70 \\
\hline
\end{tabular}

$\mathrm{DN}=$ Diámetro Normal; $\mathrm{CD}=$ Categoría diamétrica.

$\uparrow$ Para estos árboles pequeños, la biomasa se obtuvo de forma directa.

${ }^{\dagger}$ El porcentaje de humedad se determinó con base en el promedio obtenido del fuste, ramas y follaje.

La distribución relativa de la biomasa entre los componentes de los árboles de Abies religiosa, fue como sigue: en el fuste varió de 64.6 a $88.3 \%$, con un promedio de $81.3 \%$; en el tocón el porcentaje varió de 1.7 a $7.9 \%$, con un promedio de $3.3 \%$; en las ramas la variación fue de 0 (en un árbol las ramas eran tan pequeñas que se consideraron como follaje) a $11.3 \%$, con un promedio de $6.9 \%$; la variación del follaje fue de 3.5 a $20.2 \%$, con un promedio de $8.6 \%$.
La ecuación que relacionó la biomasa en función del diámetro normal tuvo un coeficiente de determinación $\left(\mathrm{R}^{2}\right)$ de 0.99. Los parámetros del modelo y la dispersión de puntos de los valores de biomasa observados se presentan en las Figura 3. 

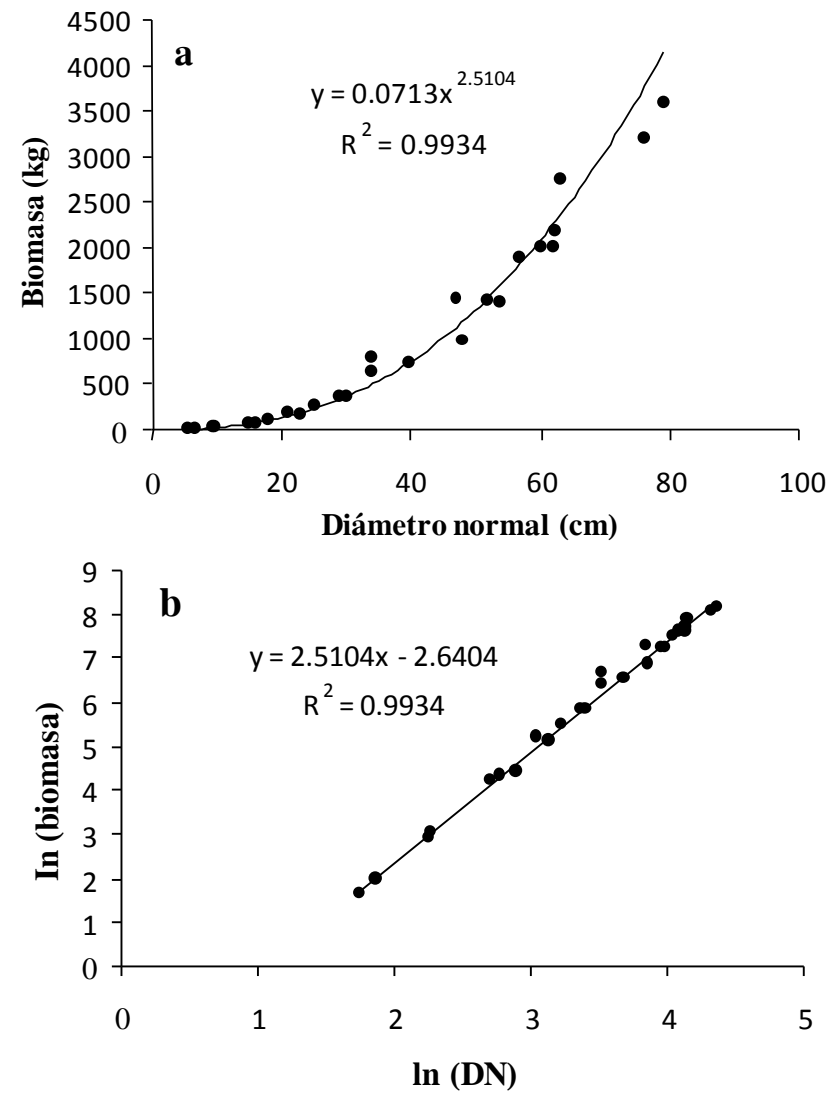

Figuras 3. Dispersión de los valores observados para estimar la biomasa en Abies religiosa Schl. et Cham., y la línea de regresión generada con el modelo potencial (a) y modelo linerizado (b). DN = Diámetro normal.

Little y Jackson (1976) recomiendan linearizar el modelo $\mathrm{Y}=b \mathrm{X}^{k}$ para facilitar la interpretación del análisis de regresión; ello se logra mediante la transformación logarítmica indicada en la Ec. 2.

Tanto el modelo en su forma original (Ec. 3) como el linearizado (Ec. 4) fueron confiables, por lo que se consideró suficiente utilizar el diámetro normal (DN) para estimar la biomasa aérea en árboles de Abies religiosa (Figuras 3a y 3b).

$\mathrm{B}=0.0713 \mathrm{DN}^{2.5104}$

(Ec. 3)

$\operatorname{Ln}(B)=-2.6404+2.5104 \ln (\mathrm{DN})$

\section{Estimación del carbono capturado por Abies religiosa}

En al contenido de $\mathrm{C}$ no se encontraron diferencias estadísticas $(\mathrm{P} \leq 0.05)$ entre partes del árbol ni entre muestras (repeticiones) de cada parte. Por ello se calculó un factor promedio de todo el árbol, el cual se usó para estimar el contenido de carbono en Abies religiosa, y fue de $46.48 \%$, factor que se aplicó a los árboles muestreados (Cuadro 2).
Cuadro 2. Porcentajes de carbono de cada parte del árbol determinados en el laboratorio.

\begin{tabular}{cccc}
\hline Número de muestra & Follaje & Ramas & Fuste \\
\hline 1 & 46.69 & 46.07 & 45.87 \\
2 & 47.77 & 45.63 & 46.50 \\
3 & 45.94 & 45.92 & 45.42 \\
4 & 45.92 & 46.81 & 46.78 \\
5 & 45.15 & 46.12 & 46.56 \\
6 & 44.94 & 46.09 & 46.73 \\
7 & & 46.17 & 46.71 \\
8 & & 45.1 & 46.65 \\
9 & & 45.95 & 47.71 \\
10 & & 46.14 & 46.72 \\
Promedios & $\mathbf{4 6 . 0 6}$ & $\mathbf{4 6 . 0 0}$ & $\mathbf{4 6 . 5 6}$ \\
\hline
\end{tabular}

Con los contenidos de carbono de los 26 árboles muestreados, se obtuvieron las ecuaciones alométricas correspondientes para estimar este elemento con base en el diámetro normal, ecuación que se ajusto al modelo $\mathrm{Y}=$ $b \mathrm{X}^{k}$. La dispersión de datos y la línea ajustada se muestran en la Figura 4a. Lo mismo se hizo con la forma linearizada (Ec. 2), y los datos observados y predichos se muestran en la Figura 4b.
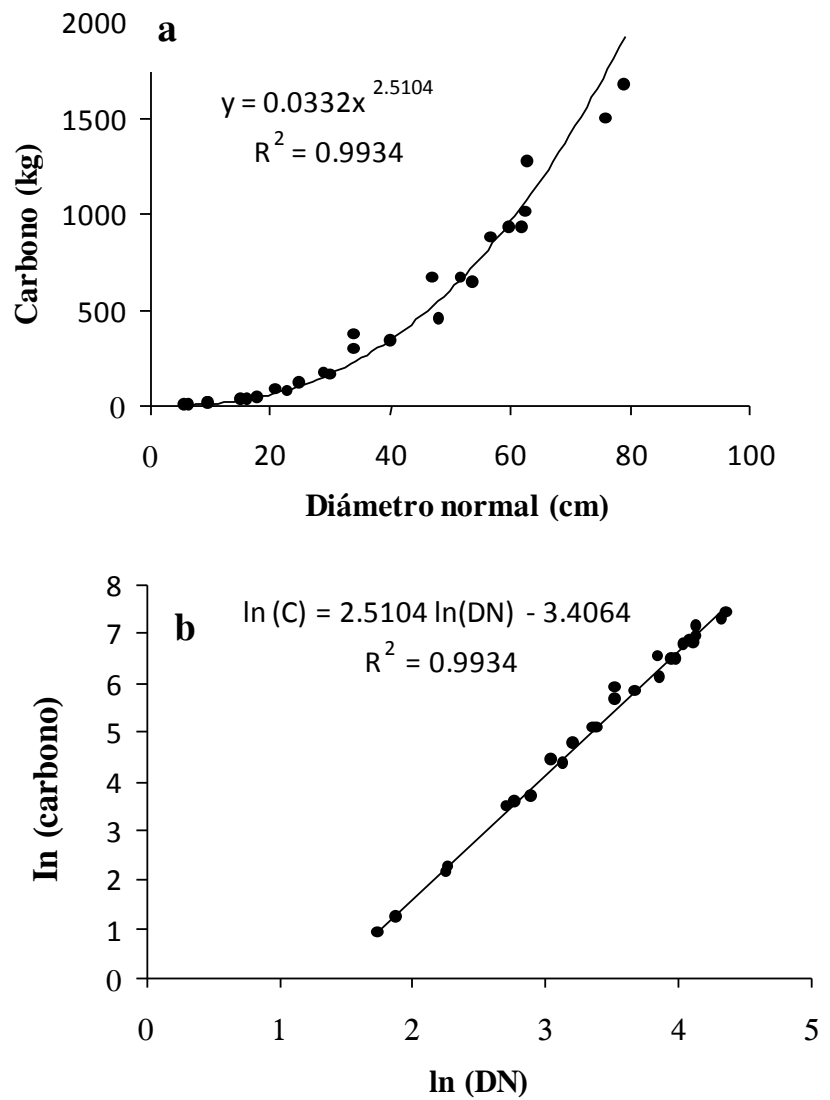

Figuras 4. Dispersión de los valores observados para estimar el carbono en Abies religiosa Schl. et Cham., y la línea de regresión generada con el modelo potencial (a) y modelo linerizado (b). DN = Diámetro normal. 
Tanto en el modelo original como en el linearizado, el modelo fue significativo $(\mathrm{P} \leq 0.01)$ y el coeficiente de determinación fue de 0.99. Estos valores indican que ambos modelos son confiables para estimar el carbono de los árboles de Abies religiosa con base en el diámetro normal.

Tales ecuaciones son las siguientes:

$$
\begin{aligned}
& \mathrm{CAA}=0.0332 \mathrm{DN}^{2.5104} \\
& \ln (\mathrm{CAA})=-3.4064+2.5104 \ln (\mathrm{DN})
\end{aligned}
$$

Díaz et al. (2007) determinaron que el factor para calcular la cantidad de carbono en Pinus patula Schl. et Cham. fue de 0.5 , lo que quiere decir que $50 \%$ de la biomasa de dicha especies es carbono. En Abies religiosa fue de 0.4648 , porcentaje menor al determinado en $P$. patula. Esta diferencia se atribuye a que el pino contiene mayores contenidos de resina y de hemicelulosa que Abies (Cuadro 3), compuestos que contienen carbono.

Cuadro 3. Componentes químicos de la madera de Abies y Pinus.

\begin{tabular}{lcc}
\hline Porciento & Abies sp. & Pinus sp. \\
\hline Celulosa & 48.2 & 47.1 \\
Hemicelulosa & 20.5 & 25.2 \\
Lignina & 26.5 & 25.6 \\
Sustancias pépticas & 0.6 & 0.9 \\
Resinas & 0.0 & $2-4$ \\
Taninos, gomas & 3.7 & 1.3 \\
Cenizas & 0.66 & 0.4
\end{tabular}

Fuente: Sjostrom, 1981.

\section{CONCLUSIONES}

En árboles de Abies religiosa la mayor parte de la biomasa se ubica en el fuste $(84.5 \%)$, mientras que en ramas se concentra $6.9 \%$ y en follaje $8.6 \%$. La ecuación generada para estimar la biomasa de Abies religiosa con base en el diámetro normal, es $\mathrm{B}=0.0713 \mathrm{DN}^{2.5104}$, con un ajuste de 0.99 .

El contenido de carbono en esta especie equivale a $46.48 \%$ de la biomasa total del árbol, y esta proporción es la misma en fuste, ramas y follaje. La ecuación para estimar el carbono en Abies religiosa con base en el diámetro normal, es CAA $=0.0332 \mathrm{DN}^{2.5104}$, con un ajuste de 0.99 .

\section{BIBLIOGRAFÍA}

Acosta M M, J Vargas H, J D Etchevers B, A Velásquez M (2002) Estimación de la biomasa aérea mediante el uso de relaciones alométricas en seis especies arbóreas en Oaxaca, México. Agrociencia 6:725-736.

Castellanos B J, A Velásquez M, J Vargas H, C Rodríguez F y A M Fierros G (1996) Producción de biomasa en un rodal de Pinus patula. Agrociencia 30:123-128.

Castro Z R, R Arteaga R, M Vázquez P, J L Jiménez R (2002) Introducción a la Meteorología. Universidad Autónoma Chapingo. Chapingo, Edo. de México. 247 p.

Chapman J W, S T Gower (1991) Aboveground production and canopy dynamics in sugar maple and read oak trees in southwestern Wisconsin. Can. J. For. Res. 21:1533-1543.

Díaz F R, M Acosta M, F Carrillo A, E Buendía R, E Flores A, J D Etchevers B (2007) Determinación de ecuaciones alométricas para estimar biomasa y carbono en Pinus patula Schl. et Cham. Madera y Bosques 13:25-34.

Epstein, R, C Rogers (2004) The impacts of $\mathrm{CO}_{2}$ and climate change on public health in the Inner City. The Center for Health and the Global Environment-Harvard Medical School. Disponible en: http://www.med.harvard.edu/chge (Mayo 2006).

Figueroa N C, J D Etchevers B, A Velázquez M, M Acosta M (2005) Concentración de carbono en diferentes tipos de vegetación de la Sierra Norte de Oaxaca. Terra 23:57-64.

Gayoso A J (2002) Medición de la Capacidad de Captura de Carbono en Bosques de Chile y Promoción en el Mercado Mundial. Universidad Austral de Chile. Valdivia, Chile. 53 p.

Geron C D, G A Ruark (1988) Comparison of constant and variable allometric rations for predicting foliar biomass of various tree genera. Can. J. For. Res.18:1298-1304.

Grier C C, R H Waring (1974) Conifer foliage mass related to sapwood area. For. Sci. 20:205-206.

Huxley J S (1932) Problems of Relative Growth. The Dial Press, New York. $276 \mathrm{p}$.

Little T M, F Jackson H (1976) Métodos Estadísticos para la Investigación en la Agricultura. Ed. Trillas. México. 270 p.

NETL (2004) Developing the Technology Base and Infrastructure to Enable Sequestration as a Greenhouse Gas Mitigation Option. National Energy Technology Laboratory. U.S.A. Disponible en: http//www.netl.doe.gob (Junio 2006).

Pastor J, J D Aber, J M Melillo (1984) Biomass prediction using generalized allometric regressions for some northeast tree species. For. Ecol. Manage. 7:265-274.

Schulze E D, Ch Wirth, M. Heimann (2000) Managing forests after Kioto. Science 289:2058-2059.

Sjostrom E (1981) Wood Chemistry Fundamentals and Applications. Laboratory of Wood Chemistry Forest Products Department. University of Technology Helsinki, Finland. 223 p.

Villers R L, I Trejo V (2000) El cambio climático y la vegetación en México. In: México: Una Visión Hacia el Siglo XXI. El Cambio Climático en México. G García (comp). Ed. Toffer, S. A. de C. V. PUMA-UNAM. México, D. F. pp:63-82. 